\title{
Fluorescent optical fiber sensors for cell viability monitoring
}

\author{
Ilia Sergachev, ${ }^{a}$ Alexander Rusanov, ${ }^{a}$ Evgeny Trushkin, $^{\text {a }}$ Dmitry Sakharov, ${ }^{\text {*a }}$ \\ Uwe Marx ${ }^{\mathrm{b}}$ and Alexander Tonevitsky
}

Received 31st January 2013

Accepted 8th April 2013

DOI: 10.1039/c3an00248a

www.rsc.org/analyst
A new simple method for non-invasive cell culture viability monitoring based on vital fluorescent stains is introduced, and its efficiency for long-term experiments on cells is demonstrated. In contrast to common methods for cell viability control, which are usually either destructive (like flow-type counters or dead cells coloring and counting), or hardly quantitative like fluorescent microscopy, the method described is automated, does not require the removal of cells from their growth area and is sensitive enough to deal with as low as tens of cells.

\section{Introduction}

Human-on-a-chip systems are a new and interesting way of substance testing, and are already widely used. ${ }^{1}$ The use of such systems for non-invasive automated cell viability monitoring is especially interesting, because lots of routine human work can be eliminated when working with multiple cell cultures and substances. Most commonly, investigated cells are placed after some preparation into a closed-cycle media circulation system, where they live for a rather long time, like days and weeks. In the usual tasks, like substance testing, for human-on-a-chip systems it is very important to know the state of the cells with minimal possible influence on them.

A broad range of commercial vital stains for all visible and near-visible spectra is available for cell visualization using a fluorescent microscope: being membrane-permeable and almost non-fluorescent, they selectively bind to certain parts of the cell and become fluorescent or converted to an active state during some metabolic process and are retained inside the cell even after division. ${ }^{2,3}$ Another common way for making cells visible is using GFP-transfected cells, which usually provides more stable fluorescent signals because the concentration of the stain within the cells remains almost constant. ${ }^{4,5}$ However, transfected cells are sometimes avoided because of the unpredictable influence of the modification to the results of the experiment. Cells that die after dye activation can provide an unwanted fluorescence background for rather a long time after death. The use of a second dye of another color, which is selective for necrosis or apoptosis, usually solves this problem.

${ }^{a}$ SRC Bioclinicum, Ugreshskaya 2-85, Moscow, 115088, Russia. E-mail: d.sakharov@ bioclinicum.com

${ }^{b}$ Department of Medical Biotechnology, Technische Universität Berlin, Gustav-MeyerAllee 25, Berlin, D-13355, Germany

'Institute of General Pathology and Pathophysiology RAMS, Baltiyskaya 8, Moscow, 125315, Russia
Another solution to the problem appears when medium around the cells flows and its volume is much larger than the volume of the cells: then the fluorescent molecules from the dead cells gradually leaves the area of interest with a flow of liquid. In human-on-a-chip systems the latter condition is well suited and therefore the fluorescent signal measured can be considered proportional to the live cell count. ${ }^{6}$ If one knows the initial cell count, one can use it as a calibration point and find out the cell count at any time by comparing the current and initial fluorescence intensity.

The developed system is based on the advantages of fluorescent vital dyes, making use of them for non-invasive automated counting by adding a registration system consisting of a CCD-spectrometer, pump laser and a common fiber bundle for light delivery from laser to sample and collection of light from sample to spectrometer. Fiber probes are known to be very effective for low-light applications like fluorescence ${ }^{7-9}$ and give the registration system lots of versatility.

\section{Experimental}

\section{Cell cultivation and preparation}

Three types of cells were used during the experiments: ER-Tracker Green (Invitrogen) stained human fibroblasts; ER-Tracker Green stained HaCaT and EGFP expressing HEK293. Postnatal human fibroblasts were obtained from skin biopsies taken during facial plastic operations. Transfected HEK293 were provided by the Laboratory of Biophotonics of the Institute of Bioorganic Chemistry, Moscow. Cells were maintained in Dulbecco's Modified Eagle's Medium (DMEM) supplemented with $10 \%(\mathrm{v} / \mathrm{v})$ heat inactivated fetal calf serum at $37{ }^{\circ} \mathrm{C}$ in a humidified atmosphere of $5 \% \mathrm{CO}_{2}$ in air. $1 \%$ antibiotic solution $\left(100 \mathrm{U} \mathrm{mL}^{-1}\right.$ penicillin, $100 \mu \mathrm{g} \mathrm{mL}^{-1}$ streptomycin; GIBCO Invitrogen) was also added to the medium. Cultures were dissociated with $0.05 \%$ trypsin- $0.02 \%$ EDTA. Cells were 
seeded in Transwell permeable supports (Corning, USA) in $200 \mu \mathrm{L}$ of growth medium DMEM.

Staining with ER-Tracker was done in accordance with the manufacturer's protocol with $1 \mu \mathrm{M}$ final working concentration of dye.

\section{Experimental setup}

In all the experiments the cells were cultivated inside a Homunculus human-on-a-chip system ${ }^{\mathbf{1}}$ (SRC Bioclinicum, Moscow, Russia) (Fig. 1), where they were grown on a Transwell support inside a closed-cycle media circulation biochip. The construction of the biochip allows cells to be clearly visible from the bottom of it, where only a glass slide, a Transwell membrane (transparent in water) and about $200 \mu \mathrm{m}$ layer of medium separate the cells from the observer.

A custom Y-shaped fiber bundle of 1 meter total length was produced by A.R.T Photonics GmbH (Berlin, Germany) and consisted of 19 NIR 300/330/360 fibers, which were tightly packed on the sample side of the bundle and split into two SMA connectors with 18 and 1 fibers on the other sides for the spectrometer and pump laser respectively. A $50 \mathrm{~mW} 473 \mathrm{~nm}$ DPSS laser (Lasever, Ningbo, China) was chosen for fluorescence excitation of the green dyes, most of which have an absorption maximum near $500 \mathrm{~nm}$. For fluorescence signal collection, a CCS100 CCD-spectrometer (Thorlabs, Newton, NJ, USA) was used with an additional $515 \mathrm{~nm}$ longpass color glass filter (Thorlabs, Newton, NJ, USA) at the input port for rejection of pump laser radiation reflected from the sample (Fig. 2).

For minimal photobleaching and elimination of fluorescence intensity dependence on time, the pulsed laser mode was used. Pulses of $200 \mathrm{~ms}$ with $50 \mathrm{~mW}$ laser power were found suitable for all cell counts observed. This intensity is even enough for measurements carried out in moderate daylight conditions, i.e. a dark room or chamber is not required. However, it may be useful to get rid of external light when working with low numbers of cells. Laser pulses were

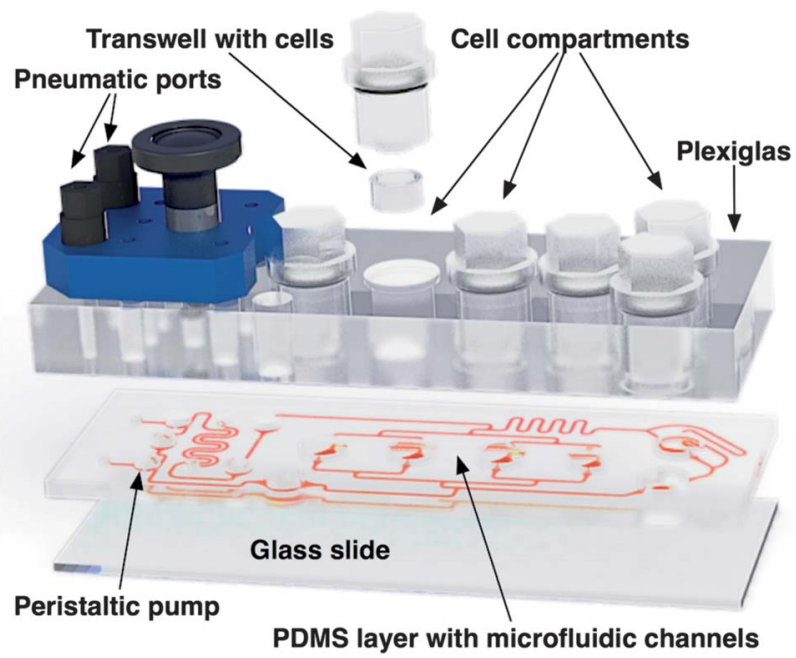

Fig. 1 Homunculus biochip exploded view.

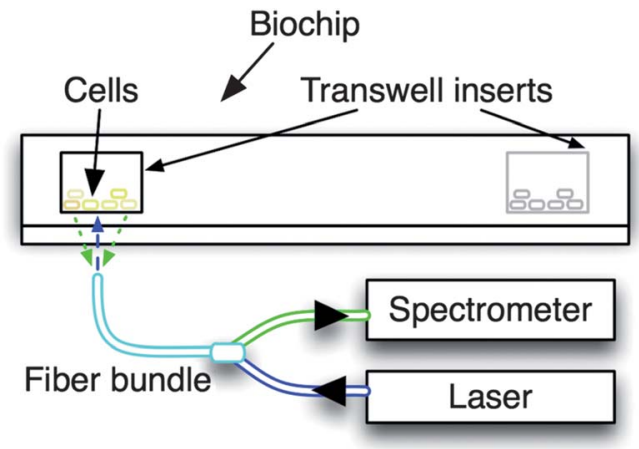

Fig. 2 Fluorescence detection scheme.

electrically synchronized to the spectrometer signal collection and were triggered manually.

The diameter of the Transwell inserts membranes we used was $4.26 \mathrm{~mm}$, and the numerical aperture of the fibers was 0.20 , therefore the optimal distance from the fiber bundle end to the cell growth area for its full coverage was around $10 \mathrm{~mm}$.

\section{Fluorescent measurements}

First, the systems sensitivity was measured. Starting from 10000 stained HaCaT cells in a 96-well plate well, spectra were recorded for the consequent amount of cells divided by 2 in 10 iterations, ending that way at about 10 cells in a well. This experiment was repeated 3 times independently with new cells and the average results were determined.

Then a long-term 2-week experiment was performed with fibroblasts. 100000 cells were placed into each of 9 Transwells, 3 of which were used as a first control group to count the initial amount of cells that seeded successfully. Then 3 of the remaining Transwells with cells were stained with ER-Tracker and the last 3 Transwells were left unstained as a second control group to find out if staining or fluorescent measurements affect cell viability. After that both the stained and unstained cells were put into Homunculus biochips and cultivated in $\mathrm{CO}_{2}$ incubator for 14 days. Every day the cell medium was replaced with a fresh one and fluorescent measurements of the stained cells were performed.

In all the experiments the background spectra were recorded from non-fluorescent cells under the same conditions.

\section{Cell imaging}

HEK293 cells were imaged at 48 hours after seeding on a Carl Zeiss AxioObserver Z1 microscope with a LD "Plan-Neofluar" $20 \times / 0.4$ objective, using Filter set 10 shift free (EX BP 450-490, BS FT 510, EM BP 515-565). Images were captured with a High Resolution Microscopy Camera AxioCam MRm and analyzed using ZEN software (Carl Zeiss, Jena, Germany).

ER-Tracker stained HaCaT cells and fibroblasts were imaged immediately after staining using the same equipment. 


\section{Colorimetric MTT assay}

The MTT [3-(4,5-dimethylthiazol-2-yl)-2,5-diphenyltetrazolium bromide, Sigma, USA] cell proliferation assay was used for measuring the number of viable cells. 20 microliters of MTT solution $\left(5 \mathrm{~g} \mathrm{~L}^{-1}\right)$ were added to the microplate wells seeded with cells. Following 4 hours incubation at $37{ }^{\circ} \mathrm{C}$ in a humidified atmosphere of $5 \% \mathrm{CO}_{2}$ in air, the MTT solution was removed. The deposited blue formazan was dissolved with $200 \mu \mathrm{L}$ of dimethyl sulfoxide (Sigma, USA). The absorbance of the solutions in the wells was measured with a plate spectrophotometer using a test wavelength of $570 \mathrm{~nm}$ and a reference wavelength of $630 \mathrm{~nm}$.

\section{Data analysis}

After acquisition each fluorescence spectrum was normalized to the corresponding background spectrum. Normalization was done so that the average intensities of the signal and background in the 700-740 $\mathrm{nm}$ interval became equal, because their difference can only be caused by optical alignment variation. Then the background spectrum was substracted from the fluorescence spectrum. The integral of resulting fluorescence spectrum in a wavelength interval corresponding to stain (490$690 \mathrm{~nm}$ ) was calculated.

A python program was written for full-cycle data processing from the acquisition of spectra for raw samples to whole longterm experiment cell viability dynamics plotting.

\section{Results and discussion}

Registered spectra from all of the fluorescent cells were recorded and compared (Fig. 3 and 4). As expected, there was no principal difference between HaCaT and fibroblasts with the same dye, but the spectrum was different for EGFP-expressing HEK293 cells. Because of the $515 \mathrm{~nm}$ long pass filter used for fluorescence detection the measured spectra were shifted to the red region in comparison to the ones known from the literature. ${ }^{10}$ The fluorescence of the cells was also confirmed by fluorescence microscopy (Fig. 4).

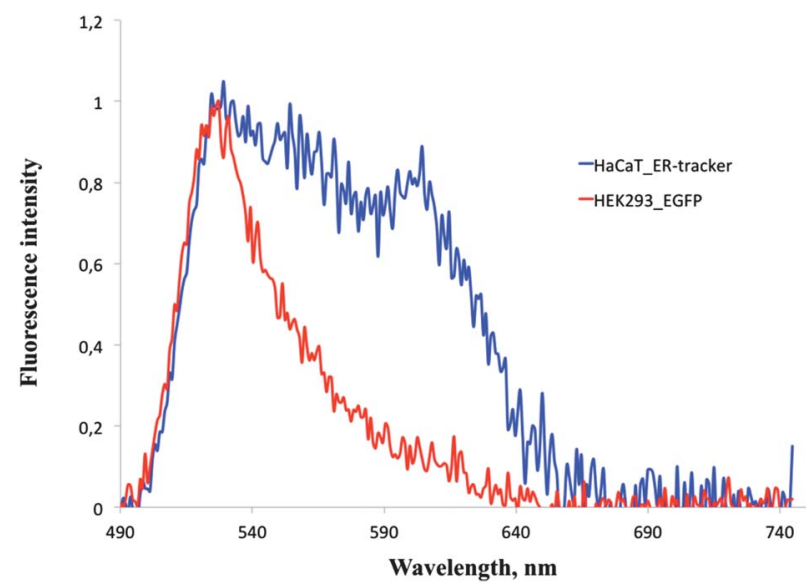

Fig. 3 Typical normalized spectra of EGFP expressing (red) or ER-Tracker stained cells (blue) after background subtraction.

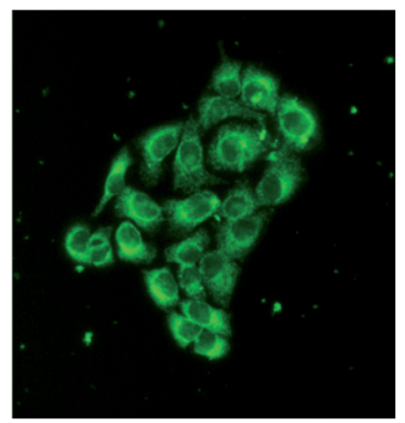

A

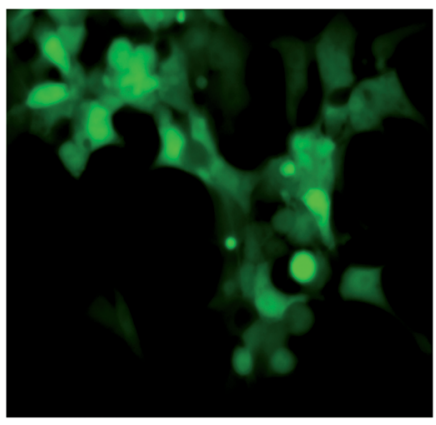

B
Fig. 4 Fluorescent images of (A) ER-Tracker stained HaCaT cells, (B) EGFPexpressing HEK293 cells.

A sensitivity test demonstrated (Fig. 5) that about 100 cells could be reliably detected by the system under the conditions described. Also the system showed perfect linearity of the measured signal dependency on cell count $\left(R^{2}=0.9998\right.$ for linear fit). The typical dispersion of measurements was around $2 \%$. Some easy improvements may be done to the system sensitivity for it to work with a lower number of cells, like use of a larger aperture spectrometer, longer acquisition times or elimination of background light.

In order to ensure the reliability of the developed method a traditional MTT approach was done to define the number of living cells. The signal was linearly proportional to the number of cells in the range from 10000 to 200 cells (data not shown). Our results indicate that the developed non-invasive cell culture viability monitoring method is as sensitive as the traditional MTT test and is capable of detecting very small numbers of viable cells.

In a 2-week experiment (Fig. 6) the gradual decrease of fluorescent signal was explained by the reduction of the total stain amount by dead cells and a moderate supply of nutrients. Cells were counted on the first day of the experiment in the first control group and on the last day in the second control group and in the fluorescent group with a cell counting chamber method, and the numbers were $95000 \pm 3000,72000 \pm 3000$ and $74000 \pm 4000$ respectively, which correlates well with the

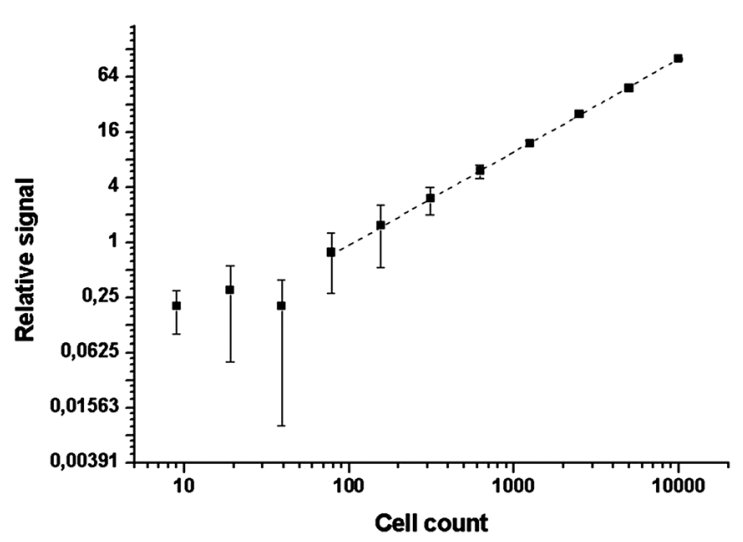

Fig. 5 System sensitivity test. 


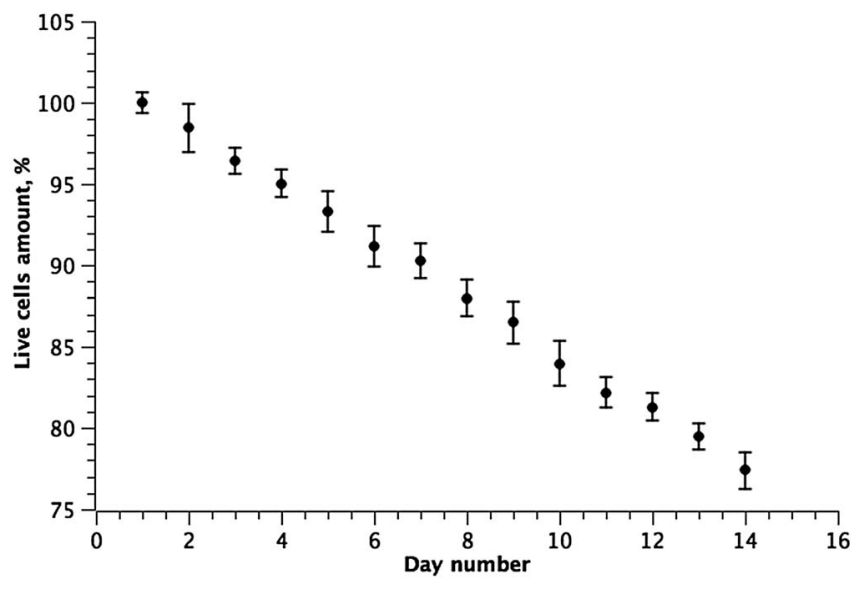

Fig. 6 Two week long monitoring of cell viability.

$77 \%$ ratio of the initial to final cell counts (Fig. 6) detected by the system developed. Also the numbers for the second control group and the fluorescent group proved the assumption that staining and fluorescent measurements do not affect cell viability.

\section{Conclusion}

A simple and highly sensitive system for automated cell viability monitoring was developed and successfully applied in a humanon-a-chip system. It is very flexible and can be adapted to various applications due to the broad range of available fluorescent markers, spectral devices, light sources and fiber solutions.

\section{Acknowledgements}

This work was supported by Russian Ministry of Science Grants no. 16.522.12.2015 and 16.552.11.7057. EGFP expressing cells were kindly provided by Konstantin A. Lukyanov, Laboratory of Biophotonics, Institute of Bioorganic Chemistry, Moscow, Russia. We would like to thank Dr Viacheslav Artyushenko for valuable scientific advice and insightful discussions.

\section{References}

1 U. Marx, H. Walles, S. Hoffmann, G. Lindner, R. Horland, F. Sonntag, U. Klotzbach, D. Sakharov, A. Tonevitsky and A. R. Lauster, ATLA, Altern. Lab. Anim., 2012, 40, 235257.

2 L. Cole, D. Davies, G. J. Hyde and A. E. Ashford, J. Microsc., 2000, 197, 239-249.

3 H. Boleti, D. M. Ojcius and A. Dautry-Varsat, J. Microbiol. Methods, 2000, 40, 265-274.

4 D. M. Chudakov, M. V. Matz, S. Lukyanov and K. A. Lukyanov, Physiol. Rev., 2010, 90, 1103-1163.

5 R. M. Hoffman, Methods Cell Biol., 85, 485-495.

6 F. Schmieder, S. Schmieder, R. Eger, S. Friedrich, A. Werner, N. Danz, U. Marx and F. Sonntag, Biomed. Eng., 2012, 57, 340-343.

7 H. Y. Choi, S. Y. Ryu, G. H. Kim, K. S. Chang, S. J. Park and B. H. Lee, Lensed Dual-Fiber Probe for the Effective Collection of Fluorescence Signals, IEEE, 2011, 23, 359361.

8 T. J. Pfefer, K. T. Schomacker, M. N. Ediger and N. S. Nishioka, Appl. Opt., 2002, 41, 4712-4721.

9 J. T. Motz, M. Hunter, L. H. Galindo, J. A. Gardecki, J. R. Kramer, R. R. Dasari and M. S. Feld, Appl. Opt., 2004, 43, 542-554.

10 I. Johnson and M. T. Z. Spence, Molecular Probes Handbook, A Guide to Fluorescent Probes and Labeling Technologies, Life Technologies, 2010. 